\title{
Tidal disruption of a super-Jupiter by a massive black hole
}

\author{
M. Nikołajuk ${ }^{1,2}$ and R. Walter ${ }^{1}$
1 ISDC Data Centre for Astrophysics, Observatoire de Genève, Université de Genève, Chemin d'Écogia 16, 1290 Versoix, Switzerland
e-mail: mrk@alpha.uwb.edu.pl; Roland.Walter@unige.ch \\ 2 Faculty of Physics, University of Bialystok, Lipowa 41, 15-424 Białystok, Poland
}

Received 30 October 2012 / Accepted 13 February 2013

\begin{abstract}
Aims. A strong, hard X-ray flare was discovered (IGR J12580+0134) by INTEGRAL in 2011, and is associated to NGC 4845, a Seyfert 2 galaxy never detected at high-energy previously. To understand what happened we observed this event in the X-ray band on several occasions.

Methods. Follow-up observations with XMM-Newton, Swift, and MAXI are presented together with the INTEGRAL data. Long and short term variability are analysed and the event wide band spectral shape modelled.

Results. The spectrum of the source can be described with an absorbed $\left(N_{\mathrm{H}} \sim 7 \times 10^{22} \mathrm{~cm}^{-2}\right)$ power law $(\Gamma \simeq 2.2)$, characteristic of an accreting source, plus a soft X-ray excess, likely to be of diffuse nature. The hard X-ray flux increased to maximum in a few weeks and decreased over a year, with the evolution expected for a tidal disruption event. The fast variations observed near the flare maximum allowed us to estimate the mass of the central black hole in NGC 4845 as $\sim 3 \times 10^{5} M_{\odot}$. The observed flare corresponds to the disruption of about $10 \%$ of an object with a mass of 14-30 Jupiter. The hard X-ray emission should come from a corona forming around the accretion flow close to the black hole. This is the first tidal event where such a corona has been observed.
\end{abstract}

Key words. galaxies: active - X-rays: galaxies - X-rays: individuals: IGR J12580+0134 - X-rays: individuals: NGC 4845

\section{Introduction}

NGC 4845 is a nearby, high surface brightness spiral galaxy with morphological type $S A(s) a b s p$ classified as a Seyfert 2 (Véron-Cetty \& Véron 2006). The distance to NGC 4845 was determined as $D=15.6 \mathrm{Mpc}$ based on the Tully-Fisher relation (Tully \& Fisher 1988). Corsini et al. (1999) obtained $D=13.1 \mathrm{Mpc}$ by including the influence of a dark matter to the systematic velocity. We adopt in this paper $D=14.5 \mathrm{Mpc}$ $\left(H_{0}=75 \mathrm{~km} \mathrm{~s}^{-1} \mathrm{Mpc}^{-1}\right)$ after Shapley et al. (2001) who performed a thorough literature search to determine the most reliable distance of this galaxy.

The galactic disk inclination is $76^{\circ}$ (Pizzella et al. 2005). Coordinates of the galaxy (i.e. $\alpha_{\mathrm{J} 2000}=12^{\mathrm{h}} 58^{\mathrm{m}} 01^{\mathrm{s}} .19$ and $\delta_{\mathrm{J} 2000}=+01^{\circ} 34^{\prime} 33^{\prime \prime}$. 02 , Véron-Cetty \& Veron 2010) set the Galactic neutral hydrogen column density into the direction of the source to $N_{\mathrm{H}}^{\mathrm{Gal}}=1.67 \times 10^{20} \mathrm{~cm}^{-2}$ (Dickey \& Lockman 1990; Stark et al. 1992).

NGC 4845 has been observed, so far, in the optical (e.g. by the Sloan Digital Sky Survey (SDSS), the Palomar Optical Sky Survey (POSS), and the Hubble Space Telescope (HST)), and in the infrared domain (by the Two Micron All Sky Survey - 2MASS and the Infrared Astronomical Satellite IRAS) (Moshir et al. 1990; Spinoglio et al. 1995; Djorgovski et al. 1998; Sanders et al. 2003; Jarrett et al. 2003; Schneider et al. 2005).

The galaxy has also been observed by the Green Bank Telescope and the Very Large Array in the radio bands 1.4, 4.8, 8.4 GHz (Condon et al. 1998, 2002). The radio emission is dominated by star-forming regions (see Filho et al. 2000, their Fig. 5d) and does not reveal water maser emission (Braatz et al. 2003). Monitoring in the X-ray domain was carried out using the Imaging Proportional Counter onboard the Einstein satellite (Fabbiano et al. 1992). The authors estimated only an upper limit in the $0.2-4.0 \mathrm{keV}$ energy band of $F_{\mathrm{X}}<2.52 \times$ $10^{-13} \mathrm{erg} \mathrm{cm}^{-2} \mathrm{~s}^{-1}$.

INTEGRAL discovered a new hard X-ray source IGR J12580+0134 (Walter et al. 2011) during an observation performed in the period January $2-11,2011$, with a position (i.e. $\mathrm{RA}=194.5212 \mathrm{deg}$, Dec $=1.5738 \mathrm{deg}, \pm 2.3 \mathrm{arcmin}, \mathrm{J} 2000.0)$ consistent with that of NGC 4845. A few days later, Swift/XRT and XMM-Newton observations confirmed the association with the central regions of the Seyfert 2 galaxy. IGR J12580+0134 was detected at a peak flux $F_{2-10 \mathrm{keV}}>5.0 \times 10^{-11} \mathrm{erg} \mathrm{cm}^{-2} \mathrm{~s}^{-1}$, corresponding to a variability by a factor $>100$, which is very unusual for a Seyfert 2 galaxy.

In Sect. 2 we report the analysis of the available highenergy observations of IGR J12580+0134 performed with XMM-Newton, Swift, MAXI, and INTEGRAL. We analyse these results in Sect. 3 and discuss them in Sect. 4.

\section{High-energy observations of IGR J12580+0134}

We analysed INTEGRAL, MAXI, Swift, and XMM-Newton observations of IGR J12580+0134. A log of the pointed observations is given in Tables 1 and 2. Throughout this section, all uncertainties are given at 1 sigma confidence level.

\subsection{INTEGRAL}

INTEGRAL data were analysed using OSA software (ver. 9). We considered data from IBIS/ISGRI in the 17.3-80 keV. The average fluxes and spectrum were extracted from the mosaic images with mosaic_spec. 
Table 1. Swift and XMM-Newton observations of IGR J12580+0134.

\begin{tabular}{lccc}
\hline \hline Instrument & Obs. ID & $\begin{array}{c}\text { Start time } \\
\text { (UTC) }\end{array}$ & $\begin{array}{c}\text { Net exp. } \\
\text { (ks) }\end{array}$ \\
\hline Swift & 00031911001 & $2011-01-12 ~ 23: 59: 01$ & 3.0 \\
Swift & 00031911002 & $2011-01-1303: 26: 01$ & 2.1 \\
XMM-Newton & 0658400601 & $2011-01-2216: 23: 28$ & 14.0 \\
Swift & 00031911003 & $2012-06-2907: 56: 59$ & 3.3 \\
\hline
\end{tabular}

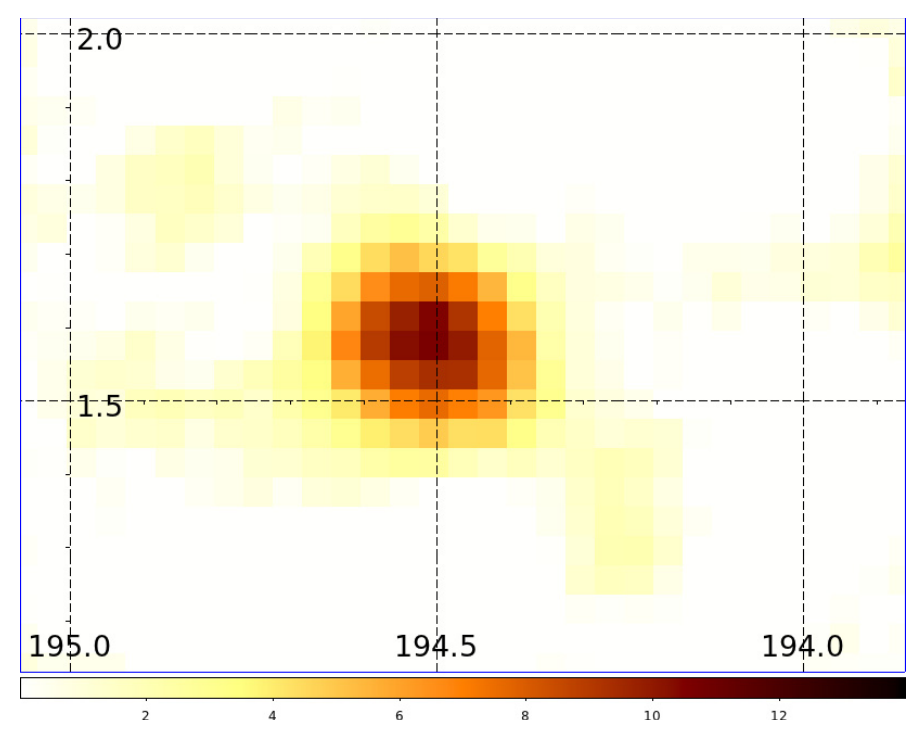

Fig. 1. The INTEGRAL IBIS/ISGRI mosaic of IGR J12580+0134 (17.3-80 keV, significance map) observed 2-11 January 2011. The dash black grid denotes equatorial J2000.0 coordinates in degrees.

INTEGRAL observations are divided into pointings with duration of $\sim 2-3 \mathrm{ks}$, which are collected during the satellite revolutions. We considered in this paper all available (i.e. 555) pointings from the discovery observation to July 21, 2011, included in revolutions 1004-1070.

Figure 1 shows the IBIS/ISGRI (Lebrun et al. 2003; Ubertini et al. 2003) mosaic obtained around IGR J12580+0134 (17.3-80 keV, significance map) accumulated from January 2 to 11,2011 . During this period the source significance reached 9.7 sigma in the $20-40 \mathrm{keV}$ energy band, corresponding to a flux of $F_{20-40 \mathrm{keV}}=(2.5 \pm 0.3) \times 10^{-11} \mathrm{erg} \mathrm{cm}^{-2} \mathrm{~s}^{-1}$ for an exposure time of $400 \mathrm{ks}$. The source was also detected by JEM-X (Lund et al. 2003) (3-20 keV) for an effective exposure time of $13 \mathrm{ks}$ with a flux of $F_{3-10 \mathrm{keV}}=(5.0 \pm 1.4) \times 10^{-11} \mathrm{erg} \mathrm{cm}^{-2} \mathrm{~s}^{-1}$.

We also analysed all publicly available IBIS/ISGRI data obtained on the field from the beginning of the mission up to January 27, 2011 using the HEAVENS interface (Walter et al. 2010) with OSA9. The INTEGRAL observations performed in previous years (i.e. from 2003 to 2010) did not reveal any hard $\mathrm{X}$-ray activity, and we obtained a five-sigma upper limit of $5.9 \times 10^{-12} \mathrm{erg} \mathrm{cm}^{-2} \mathrm{~s}^{-1}$ in the $20-40 \mathrm{keV}$ energy band.

The resulting count rates are listed in Table 3 and plotted in Fig. 2.

\subsection{MAXI}

NGC 4845 has been observed by the Monitor of All-sky X-ray Image (MAXI), attached to the Japanese Experiment Module onboard the International Space Station. Among the two X-ray instruments onboard MAXI, we analysed only the Gas Slit

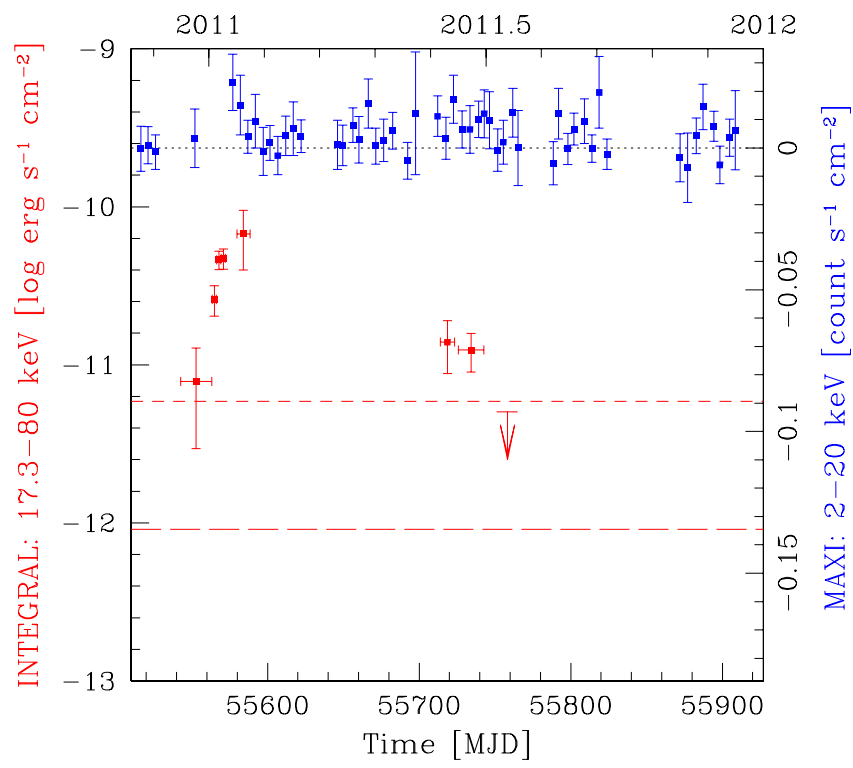

Fig. 2. The light curve of IGR J12580+0134 observed in the $17.3-80 \mathrm{keV}$ energy band by INTEGRAL/ISGRI (red points) and in the $2-20 \mathrm{keV}$ band by MAXI (blue points). The arrow shows an upper limit. Red short-dash line indicates the $5 \sigma$ upper limit of the source observed by IBIS/ISGRI before 2011. Red long-dash line shows an upper limit observed by Einstein (Fabbiano et al. 1992) and interpolated to $17.3-80 \mathrm{keV}$. The interpolation is based on the model fitted to the XMM-Newton/INTEGRAL observation. The interpolated Einstein flux is very likely overestimated because of the harder spectrum observed in 2011.

Camera (GSC) data in the present paper, because of its higher sensitivity (Matsuoka et al. 2009; Mihara et al. 2011).

Figure 2 shows the MAXI light curve of NGC 4845 in the 2-20 keV band, obtained from the MAXI/GSC on-demand process interface with the source and background radii of 2 and $3 \mathrm{deg}$, respectively, centred on the galaxy.

\subsection{Swift}

The field of view (FOV) around IGR J12580+0134 was observed three times with Swift/XRT on January 12 and 13, 2011 and on June 29, 2012 for a total exposure of 3.1, 2.1, $3.3 \mathrm{ks}$ (observations ID 00031911001, 00031911002, and 00031911003, PI Walter), respectively. We analysed data collected in photoncounting mode (PC) by using standard procedures (Burrows et al. 2005) and the latest calibration files. The Swift/XRT "enhanced" position of the source is $\alpha_{\mathrm{J} 2000}=194.50492 \mathrm{deg}$ and $\delta_{\mathrm{J} 2000}=1.57579 \mathrm{deg}$ with the error of 2.1 arcsec. To obtain those values we followed the procedure described in Evans et al. (2009) where we used the Swift/XRT-Swift/UVOT alignment and matching UVOT FOV to the USNO-B1 catalogue. We extracted light curves and spectra of IGR J12580+0134 using the Level 2 event files ${ }^{1}$. No pile-up problems were found in either observation. Therefore, we used circle region with radius of 20 pixels ( $\sim 47$ arcsec) around the source coordinates. The background region, which lies in the no visible sources area, was also chosen as a circle with a radius of 50 pixels. There are a number of bad columns on the XRT CCD, and the corresponding pixels were not used to collect the data. To account for them, we used a corrected exposure map created with the XRTEXPOMAP command

\footnotetext{
1 The online XRT analysis threads http://www . swift . ac . uk/XRT . shtml
} 
Table 2. INTEGRAL observations of IGR J12580+0134.

\begin{tabular}{|c|c|c|c|c|}
\hline \multirow[t]{2}{*}{ Revolutions } & \multicolumn{2}{|c|}{ Start time } & \multirow{2}{*}{$\begin{array}{l}\text { Stop time } \\
\text { (UTC) }\end{array}$} & \multirow{2}{*}{$\begin{array}{r}\text { EXP } \\
(\mathrm{ks}) \\
\end{array}$} \\
\hline & (UTC) & (MJD) & & \\
\hline 1004-1006 & 2011-01-02 13:51:14 & 55563.57725 & 2011-01-11 04:47:40 & 100.0 \\
\hline 1009 & 2011-01-18 19:09:42 & 55579.79841 & 2011-01-22 01:49:00 & 1.6 \\
\hline 1012 & 2011-01-27 10:55:12 & 55588.45500 & 2011-01-27 11:21:46 & 1.0 \\
\hline 1052-1055 & 2011-05-28 00:16:37 & 55709.01155 & 2011-06-06 03:15:17 & 44.2 \\
\hline 1057-1061 & 2011-06-10 03:14:24 & 55722.13500 & 2011-06-24 03:55:16 & 90.4 \\
\hline 1063 & 2011-06-28 12:02:32 & 55740.50176 & 2011-06-30 17:10:01 & 29.0 \\
\hline $1067-1068$ & 2011-07-10 21:03:15 & 55752.87726 & 2011-07-15 16:05:07 & 45.0 \\
\hline 1070 & 2011-07-20 08:31:40 & 55762.35533 & 2011-07-21 16:29:40 & 11.7 \\
\hline
\end{tabular}

Notes. EXP indicates the effective exposure time.

Table 3. Intensities of IGR J12580+0134 observed by INTEGRAL/ ISGRI.

\begin{tabular}{|c|c|c|c|}
\hline $\begin{array}{l}\text { Start time } \\
\text { (MJD) }\end{array}$ & $\begin{array}{l}\text { End time } \\
\text { (MJD) }\end{array}$ & $\begin{array}{l}\text { Count rate } \\
\left(\text { count s }^{-1}\right)\end{array}$ & Signific. \\
\hline \multicolumn{4}{|c|}{ During flare (i.e. Jan. 2011) } \\
\hline $55542.63936 \quad a$ & 55563.22030 & $0.192 \pm 0.120$ & 1.6 \\
\hline 55563.57725 & 55566.23899 & $0.635 \pm 0.139$ & 4.6 \\
\hline 55566.56943 & 55569.20899 & $1.132 \pm 0.153$ & 7.4 \\
\hline 55569.56013 & 55572.19978 & $1.157 \pm 0.167$ & 6.9 \\
\hline 55579.79841 & 55588.47273 & $1.652 \pm 0.674$ & 2.4 \\
\hline \multicolumn{4}{|c|}{ During flare - total } \\
\hline 55563.57725 & 55572.19978 & $0.944 \pm 0.087$ & 10.8 \\
\hline \multicolumn{4}{|c|}{ After flare (i.e. Jun.-Jul. 2011) } \\
\hline 55713.98265 & 55723.36030 & $0.341 \pm 0.125$ & 2.7 \\
\hline 55725.96026 & 55742.71529 & $0.304 \pm 0.083$ & 3.7 \\
\hline 55752.87726 & 55763.68727 & $0.013 \pm 0.111$ & 0.1 \\
\hline \multicolumn{4}{|l|}{ After flare - total } \\
\hline 55713.98265 & 55763.68727 & $0.269 \pm 0.056$ & 4.8 \\
\hline \multicolumn{4}{|c|}{ Period < 2011 (Jan. 2003-Dec. 2010) } \\
\hline $52644.50424 \quad a$ & 55545.30596 & $0.014 \pm 0.026$ & 0.5 \\
\hline \multicolumn{4}{|c|}{ Period > 2011 (Jan.-Jul. 2011) } \\
\hline 55563.57724 & 55763.68724 & $0.470 \pm 0.047$ & 9.9 \\
\hline
\end{tabular}

Notes. ${ }^{(a)}$ Data obtained through the HEAVENS interface.

and used it to create the ancillary response files with the command xrtmkarf. We maximised the signal-to-noise $(\mathrm{S} / \mathrm{N})$ by summing up all the available data obtained in January 2011 (effective exposure time $5116 \mathrm{~s}$ ). The final extracted spectrum was rebinned to have a minimum of 30 counts in each energy bin. Only 12 source photons were detected in June 2012.

\subsection{XMM-Newton}

XMM-Newton observed IGR J12580+0134 on January 22, 2011 (obs. ID 0658400601, total exposure time $\sim 21 \mathrm{ks}$, PI Walter) and obtained a source position of $\alpha_{\mathrm{J} 2000}=194.50427 \mathrm{deg}$ and $\delta_{\mathrm{J} 2000}=1.57585 \mathrm{deg} 4.1 \operatorname{arcsec}$ (Fig. 3).

During this observation all the EPIC-pn and EPIC-MOS cameras were operated in full frame mode. The galaxy was observed with both the EPIC-pn and EPIC-MOS; however, in this paper we focus on the data obtained from the first detector.

We processed XMM-Newton observation data files (ODFs) with pipeline EPROC (Science Analysis System, SAS, v.10.0) in order to produce a calibrated event list. The event file was filtered to exclude high background time intervals following the

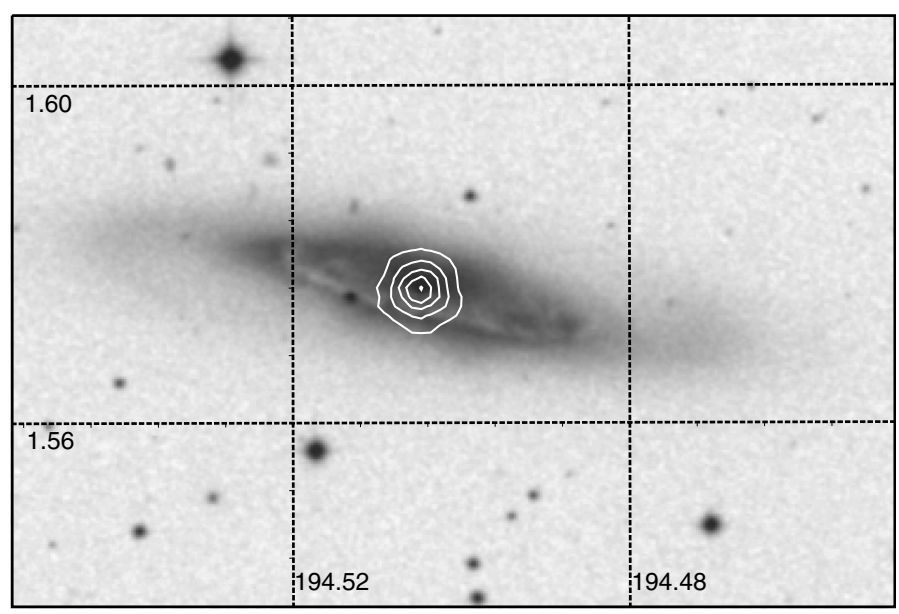

Fig. 3. The optical finding chart of NGC 4845 taken from the Space Telescope Science Institute - Digitized Sky Survey (STSciDSS). White solid contours show the XMM-Newton Epic-pn image of IGR J12580+0134 (0.8-10 keV, significance map). The black grid denotes equatorial $\mathbf{J} 2000.0$ coordinates in degrees.

recommendations of the SAS analysis thread ${ }^{2}$. We extracted the light curve in the 10-12 keV energy band using all field of view (FOV). We excluded from further analysis time intervals during which the count rate in the $10-12 \mathrm{keV}$ energy band was higher than 14.0 count $\mathrm{s}^{-1}$. The resulting effective exposure time was $14.02 \mathrm{ks}$. We extracted spectra and relevant light curves in the $0.1-2,2-10$, and $0.1-10 \mathrm{keV}$ energy bands. Since the X-ray count rate of IGR J12580+0134 is high, we corrected the product for pile-up extracting the source in an annulus with an inner radius of 1.25 pixels. The background extraction region was chosen at $\sim 9.5$ arcmin from NGC 4845 , far away from any sources but still in the same CCD of the EPIC-pn camera. All EPIC images and spectra were corrected for Out-of-Time (OoT) events. EPIC-pn spectra were rebinned before fitting so as to have at least 200 counts per energy bin.

\section{Data analysis and results}

\subsection{Source spectrum}

We extracted the XMM-Newton Epic-pn light curves in the $0.1-2 \mathrm{keV}$ (Soft) and 2-10 keV (Hard) energy bands with time bins of $10 \mathrm{~s}$. These two light curves (rebinned to $100 \mathrm{~s}$ ) and the hardness ratio $H R=($ Hard - Soft $) /($ Hard + Soft $)$ are shown

\footnotetext{
2 How to extract PN spectra see also http://xmm.esa.int/sas/ current/documentation/threads/PN_spectrum_thread.shtml
} 


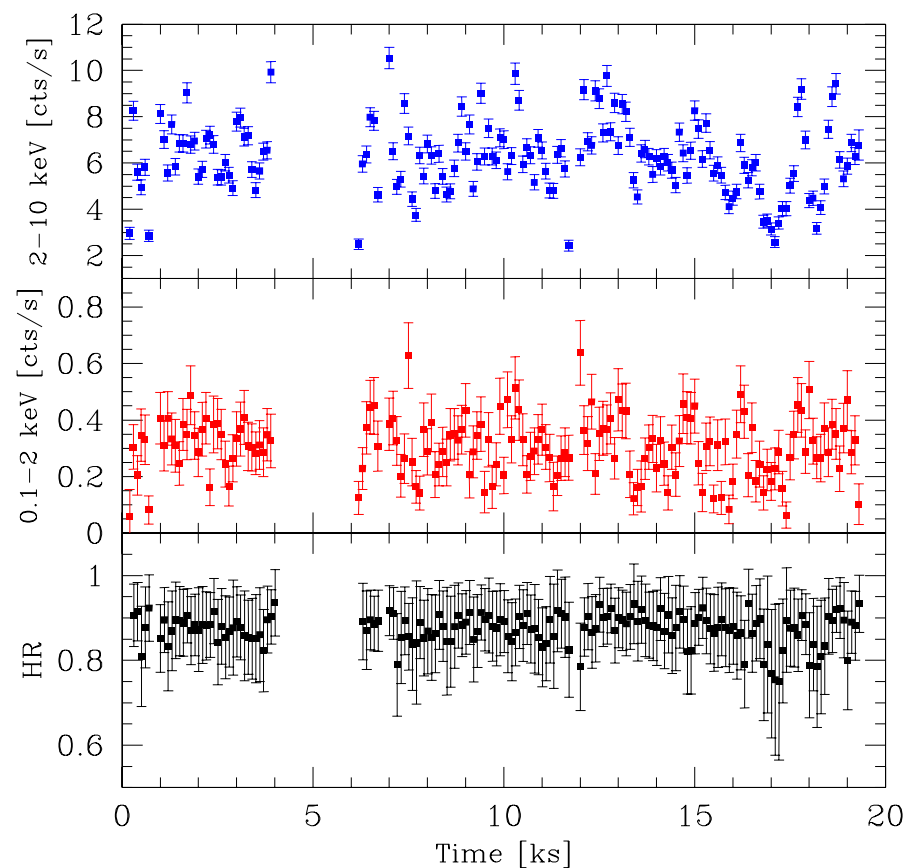

Fig. 4. XMM-Newton Epic-pn background-subtracted light curves of IGR J12580+0134. Top and middle panels show light curves extracted in the energy bands $0.1-2 \mathrm{keV}$ (Soft) and 2-10 keV (Hard), respectively. The hardness ratio, defined as HR $=($ Hard - Soft $) /($ Hard + Soft $)$, is reported in the bottom panel. The time bin is $100 \mathrm{~s}$.

in Fig. 4, where a short time-scale variability is clearly visible. The variability time scale $\tau$ between two points characterised by the highest difference in their count rates in $2-10 \mathrm{keV}$ is equal to $90 \pm 5 \mathrm{~s}\left(\Delta \mathrm{Ampl}=10.5 \pm 2.0\right.$ count $^{-1}$, found between 17 and $19.5 \mathrm{ks}$ in Fig. 5). The count rate in the $2-10 \mathrm{keV}$ band is 6.33 count s$^{-1}$, in average, with an observed standard deviation of 2.02 count s$^{-1}$. It varies between $1.08 \pm 0.48$ and $15.33 \pm 1.77$ count $^{-1}$.

The hardness ratio does not show any significant variability suggesting that the spectral shape does not vary with flux on short time scales. To check this we extracted two spectra by selecting time intervals in which the count rate in the $2-10 \mathrm{keV}$ energy band is $<6.3$ and $>6.3$ count $^{-1}$. The first spectrum (with the effective exposure time $5.9 \mathrm{ks}$ ) could be fitted by an absorbed power law. The resulting spectral parameters are $N_{\mathrm{H}}=(7.43 \pm 0.14) \times 10^{22} \mathrm{~cm}^{-2}$ and $\Gamma=2.32 \pm 0.04$ for a $2-10 \mathrm{keV} X$-ray flux of $4.95 \times 10^{-11} \mathrm{erg} \mathrm{cm}^{-2} \mathrm{~s}^{-1}$. The spectrum extracted at a higher count rate (effective exposure time $8.1 \mathrm{ks}$ ) could be described using the same model, providing $N_{\mathrm{H}}=(7.11 \pm 0.10) \times 10^{22} \mathrm{~cm}^{-2}$ and $\Gamma=2.13 \pm 0.03$ for an $2-10 \mathrm{keV} X$-ray flux of $6.94 \times 10^{-11} \mathrm{erg} \mathrm{cm}^{-2} \mathrm{~s}^{-1}$.

These spectral parameters indicate that the source spectrum did not change significantly; therefore, we also extracted the average spectrum of the complete XMM-Newton observation (Fig. 6, bottom) that can be represented by the parameters $N_{\mathrm{H}}=(7.21 \pm 0.08) \times 10^{22} \mathrm{~cm}^{-2}$ and $\Gamma=2.19 \pm 0.03$. The average $2-10 \mathrm{keV} \mathrm{X}$-ray flux is $6.09 \times 10^{-11} \mathrm{erg} \mathrm{cm}^{-2} \mathrm{~s}^{-1}$ (see also Table 4).

Swift observed IGR J12580+0134 almost one week earlier (i.e. on January, 13). We performed a similar analysis to the one for XMM-Newton and found consistent results (Fig. 6, upper panel). The extracted 1-10 keV spectrum could be represented by $N_{\mathrm{H}}=(6.53 \pm 0.38) \times 10^{22} \mathrm{~cm}^{-2}$ and $\Gamma=2.36 \pm 0.14$ for a 2-10 keV X-ray flux of $4.97 \times 10^{-11} \mathrm{erg} \mathrm{cm}^{-2} \mathrm{~s}^{-1}$.

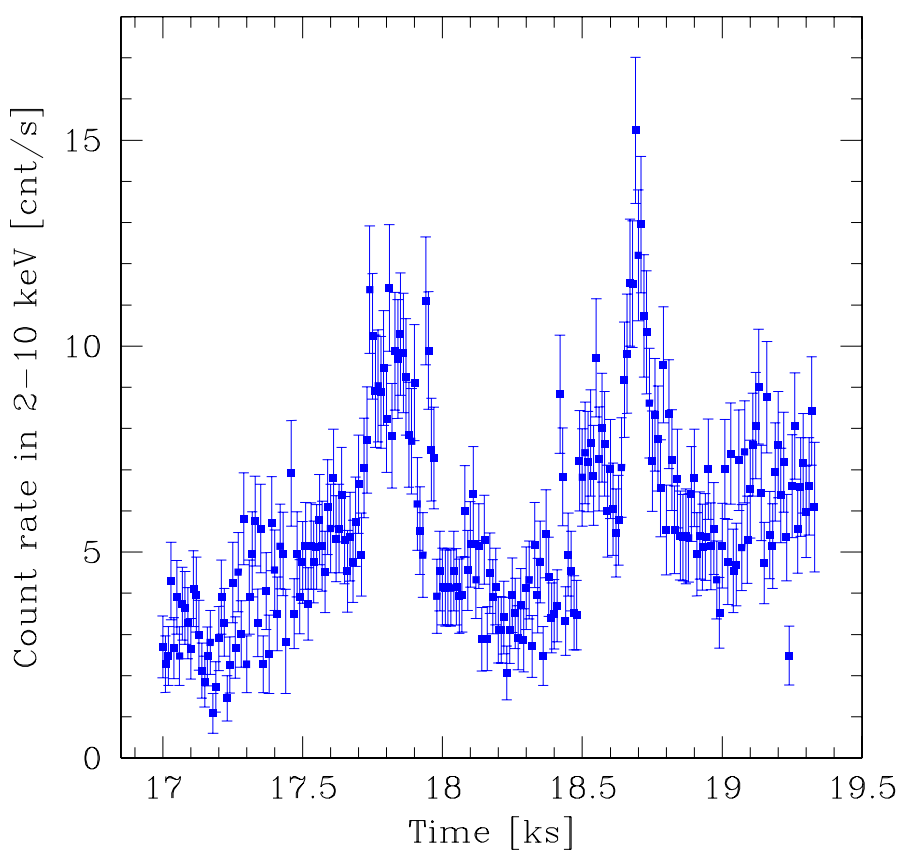

Fig. 5. Zoom of the XMM-Newton Epic-pn background-subtracted light curve showing the most variable part. The time bin is $10 \mathrm{~s}$.

The IBIS/ISGRI spectrum of the source in the $17.3-80 \mathrm{keV}$ energy bands was extracted from observations performed January 2-11 (effective exposure of $100 \mathrm{ks}$ ). The $17.3-80 \mathrm{keV}$ spectrum could be fitted $\left(\chi^{2}\right.$ by d.o.f. $\left.=0.40\right)$ by using a powerlaw model with $\Gamma=2.17$ for an average hard X-ray flux of $F(17.3-80 \mathrm{keV})=5.44 \times 10^{-11} \mathrm{erg} \mathrm{cm}^{-2} \mathrm{~s}^{-1}$. A common fit of the ISGRI and XMM-Newton data (with free normalisation) could be obtained, leading to $N_{\mathrm{H}}=(7.39 \pm 0.10) \times 10^{22} \mathrm{~cm}^{-2}$, $\Gamma=2.22 \pm 0.03$, and $F(17.3-80 \mathrm{keV})=(5.408 \pm 0.015) \times$ $10^{-11} \mathrm{erg} \mathrm{cm}^{-2} \mathrm{~s}^{-1}$ (Fig. 6).

\subsection{Intrinsic diffuse emission}

The XMM-Newton/Epic-pn spectrum shows an X-ray excess below $\sim 1.2 \mathrm{keV}$ (Fig. 6), which can be fitted by a black body absorbed by the Milky Way leading to a temperature of $T=$ $0.33 \pm 0.04 \mathrm{keV}$ and a flux of $F_{\text {bbody }}(0.01-10 \mathrm{keV})=1.12 \times$ $10^{-13} \mathrm{erg} \mathrm{cm}^{-2} \mathrm{~s}^{-1}$. This black body is not a physical model but does provide a good representation of the excess. Its flux is in good agreement with the diffuse emission radiated by galaxies (Bogdán \& Gilfanov 2011; Jia et al. 2012), and not very far from the Einstein upper limit and to the residual emission detected by Swift XTE in June $2012\left(F_{\text {bbody }}(0.5-2 \mathrm{keV})=8 \times\right.$ $10^{-14} \mathrm{erg} \mathrm{cm}^{-2} \mathrm{~s}^{-1}$ ).

This soft excess is completely unrelated to the nuclear flaring activity since the nucleus is very strongly absorbed. This emission comes from a region outside of the dusty torus of NGC 4845.

\subsection{Duration and frequency of the flare}

Because the source was still detected significantly in July 2011 by INTEGRAL, the flare probably lasted for 150 days at a level above $2 \times 10^{-11} \mathrm{erg} \mathrm{cm}^{-2} \mathrm{~s}^{-1}$. MAXI observed the source continuously, but at a lower sensitivity. Therefore the flare was just detected by MAXI close to the peak in January 2011. 
M. Nikołajuk and R. Walter: Tidal disruption of a super-Jupiter by a massive black hole

Table 4. The best-fit parameters to the Swift and XMM-Newton observations in January 2011.

\begin{tabular}{|c|c|c|c|c|c|c|}
\hline $\begin{array}{l}\text { Satellite/ } \\
T_{\text {exposure }} \\
\end{array}$ & Data & $\begin{array}{c}N_{\mathrm{H}} \\
\left(10^{22} \mathrm{~cm}^{-2}\right)\end{array}$ & $\Gamma$ & $\begin{array}{c}\text { Pl. Norm. at } 1 \mathrm{keV} \\
\left(10^{-2} \text { photons } \mathrm{keV}^{-1} \mathrm{~cm}^{-2} \mathrm{~s}^{-1}\right)\end{array}$ & $\begin{array}{c}F_{2-10 \mathrm{keV}} \\
\left(10^{-11} \mathrm{erg} \mathrm{cm}^{-2} \mathrm{~s}^{-1}\right)\end{array}$ & $\chi^{2} /$ d.o.f. \\
\hline Swift/5.1 ks & All data & $6.53 \pm 0.38$ & $2.36 \pm 0.14$ & $5.69 \pm 1.33$ & $4.968 \pm 0.130$ & $78.8 / 81$ \\
\hline$X M M / 5.9 \mathrm{ks}$ & Count rate $<6.3$ & $7.43 \pm 0.14$ & $2.32 \pm 0.04$ & $5.50 \pm 0.39$ & $4.945 \pm 0.077$ & $90.9 / 81$ \\
\hline$X M M / 8.1 \mathrm{ks}$ & Count rate $\geq 6.3$ & $7.11 \pm 0.10$ & $2.13 \pm 0.03$ & $5.54 \pm 0.28$ & $6.937 \pm 0.023$ & $193.7 / 150$ \\
\hline$X M M / 14 \mathrm{ks}$ & All data & $7.21 \pm 0.08$ & $2.19 \pm 0.03$ & $5.45 \pm 0.23$ & $6.088 \pm 0.020$ & $349.5 / 225$ \\
\hline
\end{tabular}

Notes. The fit to the $1-10 \mathrm{keV}$ energy range.
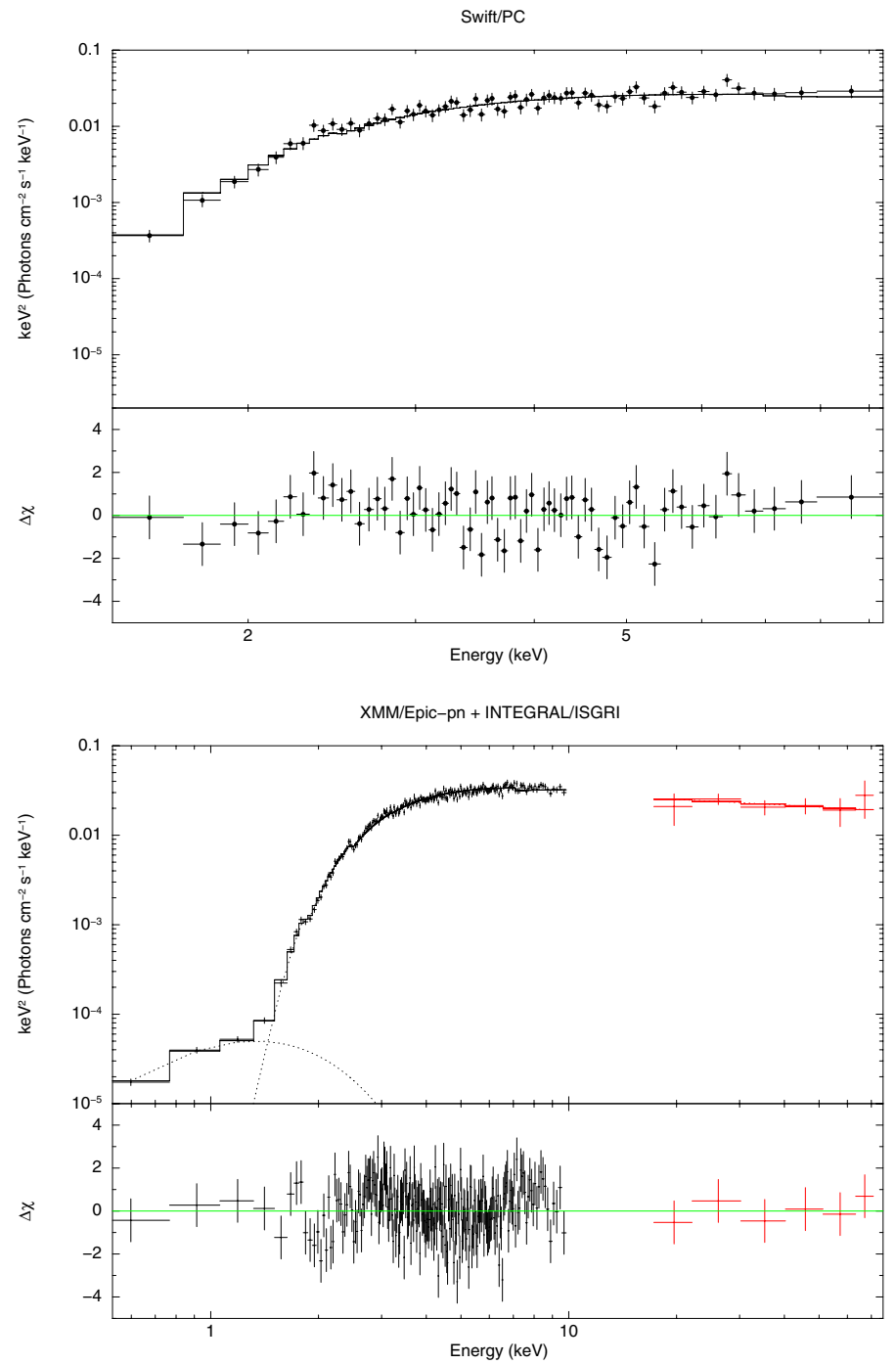

Fig. 6. Unfolded spectrum of IGR J12580+0134 from Swift observations made in PC mode (top) and unfolded XMM-Newton/Epic-pn and INTEGRAL/ISGRI spectra (bottom). The best-fit model (an absorbed power law in all spectra plus an unabsorbed black-body emission below $1 \mathrm{keV}$ in case of Epic-pn observation) is shown by solid lines in both upper panels. Lower panels indicate the residuals $(\Delta \chi)$.

Such a flare of IGR J12580+0134 with a duration of 150 days has never been detected in the past ten years by INTEGRAL, and it could have been missed only between August 2006 and December 2007 when INTEGRAL did not observe that region of the sky. The flare of NGC 4845 is therefore exceptional, and no such flare was detected for a continuous period of at least 1300 days.

\subsection{Black-hole mass}

The mass of the central black hole, $M_{\mathrm{BH}}$, of NGC 4845 can be estimated using methods based on the X-ray variability time scale.

The upper limit of $M_{\mathrm{BH}}$ can first be estimated assuming that the shortest time variability $\tau$ is related to the innermost stable circular orbit (ISCO). The XMM-Newton light curve observed in $2-10 \mathrm{keV}$ band indicates $\tau<90 \pm 5 \mathrm{~s}$. Additionally, assuming a non-rotating black hole, we can write that $\mathrm{c} \tau=R_{\mathrm{Schw}}=$ $2 G M_{\mathrm{BH}} / \mathrm{c}^{2}$. The observed variability time scale corresponds to $M_{\mathrm{BH}} \leq 9.6 \times 10^{6} M_{\odot}$.

The second technique is based on the X-ray excess variance measurements. This method uses the relationship between the black-hole mass and the X-ray variability $M_{\mathrm{BH}}=C(T-$ $2 \Delta t) / \sigma_{\mathrm{nxs}}^{2}$, where $T$ is the duration of the X-ray light curve and $\Delta t$ is the bin size, both in seconds, and $\sigma_{\mathrm{nxs}}^{2}=\sum_{i=1}^{N}\left[\left(x_{i}-\bar{x}\right)^{2}-\right.$ $\left.\sigma_{\text {err } i}^{2}\right] /\left(N \bar{x}^{2}\right)$ is the normalised excess variance (Nandra et al. 1997; Vaughan et al. 2003; O'Neill et al. 2005). We divided the 2-10 keV XMM-Newton light curve into two parts with length $T \sim 4 \mathrm{ks}$ and $\sim 10 \mathrm{ks}$ in order to avoid the gap of $5 \mathrm{ks}$ in the light curve. The estimated mean normalised excess variance, $\left\langle\sigma_{\mathrm{nxs}}^{2}\right\rangle$, of the $10 \mathrm{~s}$ bin light curve is $0.12_{-0.04}^{+0.09}$. Thus, the calculated central black hole mass in NGC 4845 is $M_{\mathrm{BH}}=2.3_{-1.0}^{+1.1} \times 10^{5} M_{\odot}$, where we assumed $C=1.42$ (i.e. that the mass of Cyg X-1 is equal to $14.8 \pm 1.0 M_{\odot}$, Orosz et al. 2011). The errors on the mass were estimated by performing Monte Carlo simulations (see details in Nikołajuk et al. 2006). The systematic errors, related to the calibration of the excess variance versus mass relationship, are larger and the black-hole mass could range in the interval $10^{4}-10^{6} M_{\odot}\left(\mathrm{O}^{\prime}\right.$ Neill et al. 2005).

\section{Discussion}

\subsection{Phenomenology}

We first consider the possibility that the observed variability is driven by absorption, i.e. that a hole opened on the line of sight in the AGN absorbing torus. The [O III] line intensity in NGC 4845 is $5.40 \times 10^{-15} \mathrm{erg} \mathrm{s}^{-1}$ (Ho et al. 1997). According to the X-ray - $[\mathrm{O} \mathrm{III}]_{\lambda 5007}$ relationship (Panessa et al. 2006) the estimated unabsorbed $2-10 \mathrm{keV}$ flux should be $\simeq 6 \times 10^{-14} \mathrm{erg} \mathrm{s}^{-1}$. However, since the X-ray flux observed in January 2011 was almost 1000 times larger (Table 4), the absorption explanation is ruled out. A strong increase in the X-ray flux, by a factor $10^{3}$, is required. We also note that the source is not Compton thick, $\left(N_{\mathrm{H}} \sim 7 \times 10^{22} \mathrm{~cm}^{-2}\right.$, see Table 4$)$, indicating that it should be bright at hard X-rays, when active.

Supernova explosions and expanding winds may emit hard X-ray flares (Immler \& Lewin 2003; Chandra et al. 2009; Saxton et al. 2012). Supernova peak X-ray luminosities have been observed up to $10^{40} \mathrm{erg} / \mathrm{s}$ with a decline usually following a $t^{-(0.5-1)}$ scaling. The peak X-ray luminosity of IGR J12580+0134 


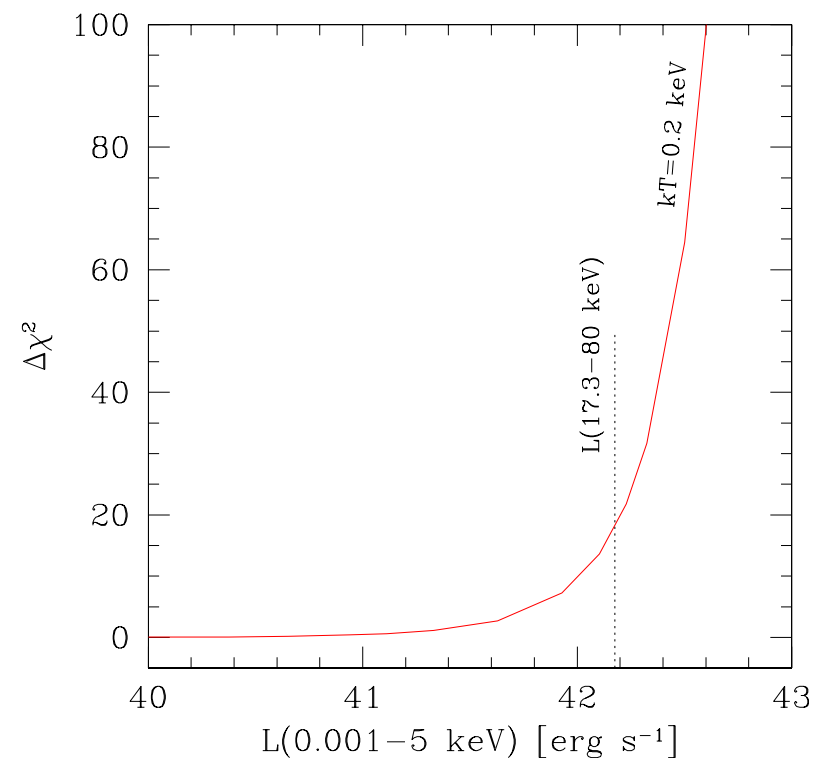

Fig. 7. Increase in $\chi^{2}$ related to an additional soft $\mathrm{X}$-ray component (black-body with temperature of $0.2 \mathrm{keV}$ ) increasing in luminosity, when fitted by the same model as used in Fig. 6. A soft X-ray component stronger than $5 \times 10^{42} \mathrm{erg} \mathrm{s}^{-1}$ should have been detected, if present.

is 100 times brighter, and it declined much faster than observed in supernovae. This, together with the position of IGR J12580+0134 at the very centre of NGC 4845, makes the supernova interpretation of the observed flare very unlikely.

A brightening of the Seyfert nucleus by a factor $10^{3}$ should therefore be explained by a sudden increase in the accretion rate. Tidal disruptions of objects by a massive black hole (see Komossa et al. 2004; Burrows et al. 2011; Cenko et al. 2012; Saxton et al. 2012) have been modelled and simulated by various authors (e.g. Rees 1988; Evans \& Kochanek 1989; Ulmer 1999; Alexander \& Kumar 2001; Alexander \& Livio 2001; Li et al. 2002; Lodato et al. 2009). The induced emission follows a power law decline with a characteristic slope of $-5 / 3$, corresponding to our observations.

The peak of the observed $17.3-80 \mathrm{keV}$ luminosity is $1.5 \times$ $10^{42} \mathrm{erg} \mathrm{s}^{-1}$. Most of luminosity of a tidal disruption event is expected to be released in the soft X-rays (and absorbed by the torus in NGC 4845). We can evaluate the maximum soft X-ray emission allowed by the data by adding a soft thermal component to the model and determine for which flux such a component would have been detected. Figure 7 shows the increase in the $\chi^{2}$ obtained depending on the soft component luminosity. For a temperature of $k T=0.2 \mathrm{keV}$, which is expected in our case (see Eq. (9) in Ulmer 1999), the soft component cannot be more than 10 times brighter than the hard X-ray component. The soft component could of course be brighter if it would peak at lower energies, which is however unexpected, especially for a low-mass black hole.

Assuming that the thermal emission is ten times brighter than the hard X-ray emission, the tidal event luminosity reached the Eddington luminosity at maximum with $L_{\text {flare }} / L_{\text {Edd }} \approx 0.6$, as observed in other tidal disruption events ( $\mathrm{Li}$ et al. 2002). The total energy radiated is then of the order of $10^{50} \mathrm{erg}$, which corresponds to the energy released by the accretion of 0.5 Jupiter $\operatorname{mass}\left(M_{\mathrm{J}}\right)$.

We fitted the decline of the IGR J12580+0134 X-ray light curve with the $\left(t-t_{\mathrm{D}}\right)^{-5 / 3}$ law where $t_{\mathrm{D}}$ is the time of the initial tidal disruption (Fig. 8). The peak luminosity of the

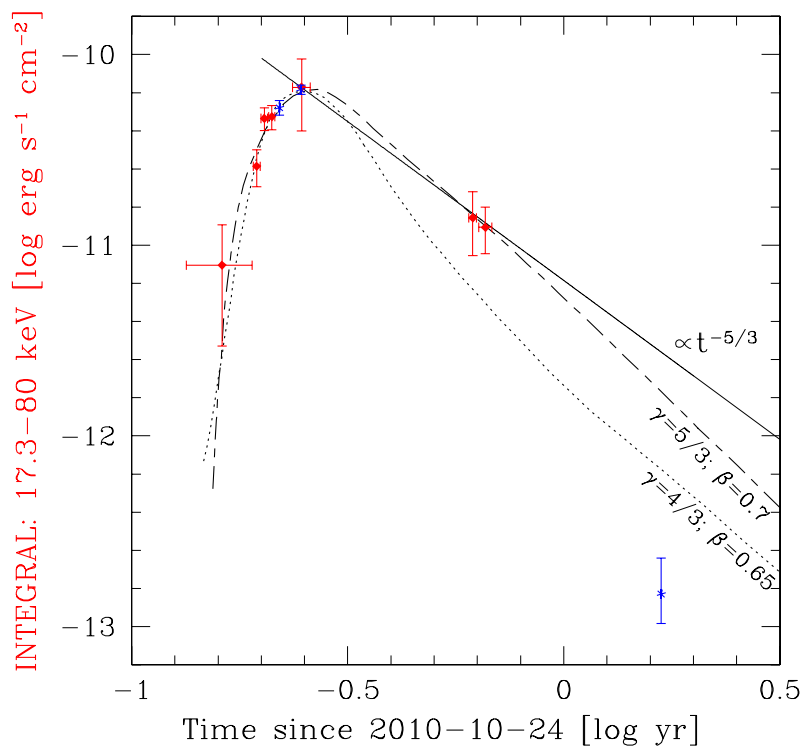

Fig. 8. The light curve of IGR J12580+0134 observed in the $17.3-80 \mathrm{keV}$ energy band. Red squared points refer to INTEGRAL data, blue crosses show Swift and XMM-Newton observations. The solid line show a model of the form $\left(t-t_{\mathrm{D}}\right)^{-5 / 3}$, as expected for fallback of material after a tidal disruption event. The long-short dash and dotted lines indicate the predictions of simulations for the disruption of a substellar object $(\gamma=5 / 3 ; \beta=0.7)$ and, respectively of a star $(\gamma=4 / 3$; $\beta=0.65$ ) (Guillochon \& Ramirez-Ruiz 2012).

flare occurred on January 22, 2011 according to MAXI and INTEGRAL. It turns out from our fit that the beginning of the tidal disruption occurred $\simeq 60-100$ days before the peak of the $\mathrm{X}$-ray flare, marking the heating of the debris at the vicinity of the black hole. A similar delay was mentioned by Li et al. (2002) as the time $\Delta t_{1}$, since the most bounded material returns to the pericentre.

\subsection{Comparison with tidal disruption simulations}

Detailed hydrodynamic simulations of tidal disruptions were performed by Guillochon \& Ramirez-Ruiz (2012) and their results parametrised. The rate of mass falling on the black hole, $\dot{M}(t)$, the time of the peak accretion, $t_{\text {peak }}$, as well the decay power-law index, $\Gamma$, depend on a few parameters: the structure and mass of the disrupted object and the minimum distance to the black hole.

We compared the simulations to our data assuming that the disrupted object is either a star or a sub-stellar object with polytropic index $\gamma$ of $4 / 3$ or $5 / 3$, respectively. We used the parametrisation included into the Appendix of Guillochon \& Ramirez-Ruiz (2012) for different impact parameters $\beta \equiv r_{\mathrm{T}} / r_{\mathrm{P}}$, where $r_{\mathrm{T}}$, and $r_{\mathrm{P}}$ are the tidal and the pericentric radii, respectively. We also assumed $M_{\star}-R_{\star}$ relations for the disrupted object valid for sub-stellar objects or stars according to Chabrier \& Baraffe (2000).

The observational constraints, $t_{\text {peak }} \simeq 0.2 \mathrm{yr}$ and the peak accretion rate $\dot{M}_{\text {peak }} \simeq 2.5 M_{\mathrm{J}} /$ yr (under the assumption of a hard X-ray radiation efficiency of $10 \%$ ), are sufficient to constrain the mass of the disrupted object. For a black-hole mass of $2.3 \times 10^{5} M_{\odot}$, the mass of the disrupted object turns out to be either $14-16 M_{\mathrm{J}}$ (for $\gamma=5 / 3$ and $\beta=0.6-1.9$ ) or $10-15 M_{\odot}$ (for $\gamma=4 / 3$ and $\beta \simeq 0.65$ ). For a black-hole mass increasing to $10^{6} M_{\odot}$, the mass of the disrupted object would be $25-28 M_{\mathrm{J}}$ (up to $75 M_{\mathrm{J}}$ for $10^{7} M_{\odot}$ ) for a sub-stellar object or 1-3 $M_{\odot}$ for 
M. Nikołajuk and R. Walter: Tidal disruption of a super-Jupiter by a massive black hole

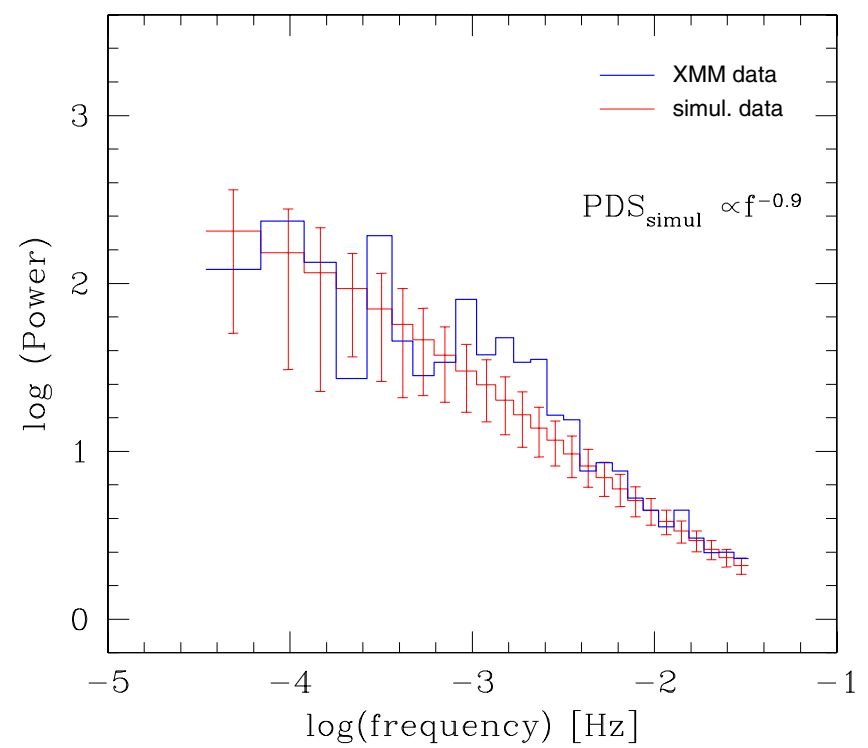

Fig. 9. The $19.1 \mathrm{ks} X M M-N e w t o n$ power spectrum (blue histogram) versus simulated 10000 powers (red histogram with error bars). The power spectrum of the simulated light curves are power law shaped with a slope of -0.9 . $1 \sigma$ error bars are shown. An excess in the observed power near the $\log$ (frequency) $=-3$ may suggest a presence of the QPO feature.

a star $(\beta \simeq 0.7)$. The $\beta$ parameter is not constrained in the case of a sub-stellar object, while a very narrow range is required to disrupt a star. The fraction of the object reaching the black hole would be very small in the case of a stellar disruption.

The hydrodynamic simulations also predict the detailed variability of the accreted mass flow with time. In the case of a star $(\gamma=4 / 3)$, less centrally condensed than a sub-stellar object, the accreted mass decreases much faster after the peak. This is illustrated in Fig. 8, where the observed flux behaves similarly to the predictions for the disruption of a sub-stellar object and contrasts with those obtained for a star.

The hydrodynamic simulations indicate, therefore, that the tidally disrupted object was probably a $14-30 M_{\mathrm{J}}$ sub-stellar object and that about $10 \%$ of its mass has been accreted on a blackhole weighting no more than $10^{6} \mathrm{M}_{\odot}$. We note that a slightly different equation of state could lead to a mass of the disrupted object as low as several $M_{\mathrm{J}}$ and that specific simulations of tidal disruption of sub-stellar objects have not yet been done.

The decline in the hard X-ray flux observed in Fig. 8 after 200-500 days can be understood as debris falling back on the remaining object core and decreasing the emission during the late evolution (see Fig. 8b in Guillochon \& Ramirez-Ruiz 2012). This would indicate that the disruption was not total. Another explanation could be that the corona disappears faster than the debris, possibly indicating a change in the geometry of the accretion flow with time.

\subsection{Power spectrum}

The power spectrum of the X-ray light curve obtained by $X M M-N e w t o n$ at the flare maximum (see Fig. 9) shows an excess in the range $0.0008-0.004 \mathrm{~Hz}$. This excess could be similar to a quasi-periodic oscillation (QPO) in the accretion flow. The frequency range of the excess corresponds to the innermost stable circular orbit for a black hole of a few $\times 10^{5} M_{\odot}$ (Remillard \& McClintock 2006).

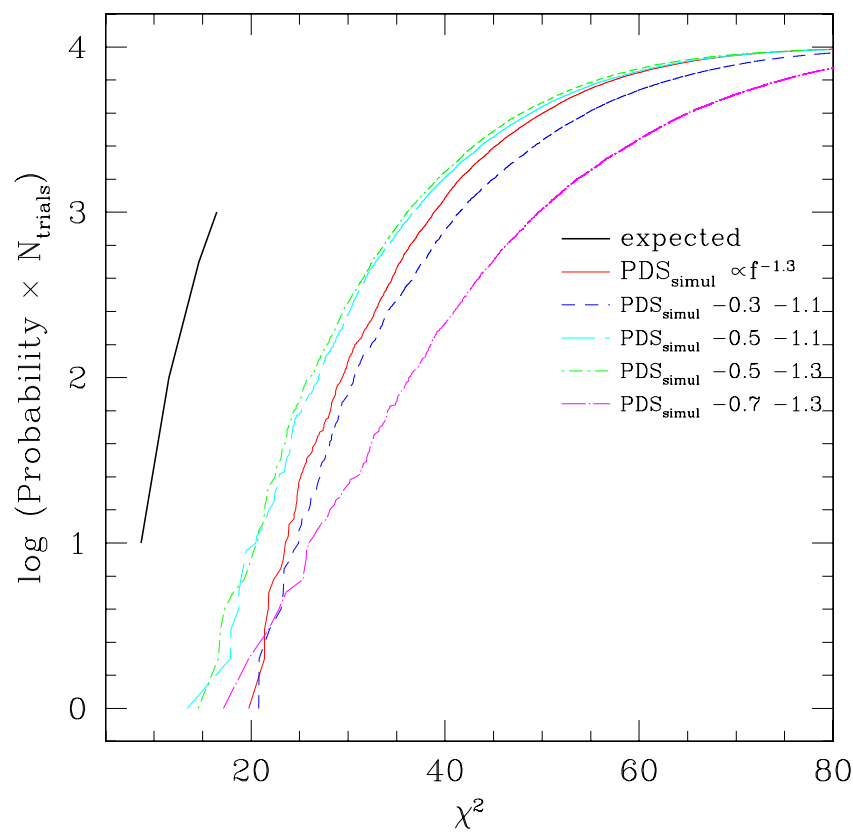

Fig. 10. Probability multiplied by number of trials $\left(N_{\text {trials }}=10000\right)$ against $\chi^{2}$ for simulated single and broken power-law models. The solid black curve points out expected probability of a perfect model. The best fit by a single power law is obtained with a slope $=-1.3$. Broken power laws with the low-frequency slope $=-0.5$, the high-frequency slope between -1.1 and -1.3 , and with broken frequency $=0.001 \mathrm{~Hz}$ provide a slightly better description of the observed power spectrum.

To investigate the significance of this excess, we performed Monte Carlo simulations. We assumed various shapes of the power spectrum of the source and generated 10000 light curves for each of them (Timmer \& Koenig 1995). The spread of the power spectra of all simulated light curves were then compared to the shape of the observed power spectrum, as outlined in Uttley et al. (2002).

We tried single power-law power spectra with slopes between -0.7 and -1.5 and broken power-law models with break frequency frozen to $10^{-3} \mathrm{~Hz}$, which differ by the low- and highfrequency slopes. Figure 10 shows the $\chi^{2}$ distributions obtained for the best models, together with the expected distribution. The deviation between the simulated and expected distribution indicates that the excess can be obtained by chance at a probability not greater than $5 \%$. We conclude that a longer XMM observation would have been very useful and note that an instrument like the Large Observatory For X-ray Timing (LOFT) would have detected several million X-ray photons during one ISCO period for this tidal event, which would have allowed a probe of the geometry of the debris falling towards the black-hole horizon.

\subsection{Tidal events frequency}

The density of stars in the centre of a well developed galaxy is around $10^{2} M_{\odot} / \mathrm{pc}^{3}$ in a region of $100 \mathrm{pc}$ in radius and is comparable to what is found in globular cluster (Marx \& Pfau 1992). These dense regions allow stellar encounters to create new gravitationally bound systems or ejections (Davies \& Benz 1995). Stars in the vicinity of a supermassive black hole may escape to infinity after encounter because their orbits are deflected (Alexander \& Kumar 2001; Alexander \& Livio 2001). Other processes may also play important roles (see brief review by Alexander 2012). The tidal event rate in bulges are estimated 
to be in the range $10^{-6}-10^{-4} \mathrm{yr}^{-1}$ galaxy ${ }^{-1}$ growing to $10^{-2}$ in case of chaotic stellar motions in a non- axisymmetric gravitational potential (Merritt \& Poon 2004). A reasonable rate for the stellar tidal disruption events is $\sim 10^{-5} \mathrm{yr}^{-1}$ galaxy ${ }^{-1}$ (Donley et al. 2002; Wang \& Merritt 2004; Gezari et al. 2009).

The discovery of a population of unbound Jupiter-mass objects has recently been mentioned by Sumi et al. (2011) and Delorme et al. (2012). The number of free-floating massive planets, which are kicked away from young star-forming regions (e.g. Quanz et al. 2010), has been estimated (Sumi et al. 2011) as one to three times higher than the number of main-sequence stars. Because of the different equations of state, the tidal radii are similar for objects of $1 M_{\odot}$ and $1 M_{\mathrm{J}}$ around a massive black hole. Tidal disruptions of planets could therefore be as frequent as star disruption. Planets could increase the rate of tidal events in galaxies a few times, but will generate lower luminosity events on average.

We estimate that INTEGRAL could detect tidal disruption flares with fluxes ten times lower than observed in IGR J12580+0134 i.e. from galaxies up to a distance of $50 \mathrm{Mpc}$, for similarly faint events. The number of galaxies within $D<$ $50 \mathrm{Mpc}$ is $\sim 5000$. The event rate detectable at hard X-rays (by INTEGRAL or Swift) could therefore be expected to be a few events every ten years. The INTEGRAL and Swift archives can be used to test these predictions.

Acknowledgements. Based on observations with INTEGRAL, an ESA project with instruments and science data centre funded by ESA member states (especially the PI countries: Denmark, France, Germany, Italy, Switzerland, Spain), and Poland and with the participation of Russia and the USA. We thank Enrico Bozzo, Laetitia Gibaud, and Claudio Ricci for help with Swift and XMM-Newton analyses; Piotr Życki for giving his software template and to Bożena Czerny for discussion about light-curve simulations. M.N. also thanks the Scientific Exchange Programme (Sciex) NMS ${ }^{\text {ch }}$ for the opportunity of working at ISDC. This research has been supported in part by the Polish NCN grants N N203 581240 and 2012/04/M/ST9/00780.

\section{References}

Alexander, T. 2012, in EPJ Web Conf., 39, 5001 Alexander, T., \& Kumar, P. 2001, ApJ, 549, 948 Alexander, T., \& Livio, M. 2001, ApJ, 560, L143

Bogdán, Á., \& Gilfanov, M. 2011, MNRAS, 418, 1901

Braatz, J. A., Wilson, A. S., Henkel, C., Gough, R., \& Sinclair, M. 2003, ApJS, 146, 249

Burrows, D. N., Hill, J. E., Nousek, J. A., et al. 2005, Space Sci. Rev., 120, 165 Burrows, D. N., Kennea, J. A., Ghisellini, G., et al. 2011, Nature, 476, 421

Cenko, S. B., Krimm, H. A., Horesh, A., et al. 2012, ApJ, 753, 77

Chabrier, G., \& Baraffe, I. 2000, ARA\&A, 38, 337

Chandra, P., Dwarkadas, V. V., Ray, A., Immler, S., \& Pooley, D. 2009, ApJ, 699,388

Condon, J. J., Cotton, W. D., Greisen, E. W., et al. 1998, AJ, 115, 1693

Condon, J. J., Cotton, W. D., \& Broderick, J. J. 2002, AJ, 124, 675

Corsini, E. M., Pizzella, A., Sarzi, M., et al. 1999, A\&A, 342, 671

Davies, M. B., \& Benz, W. 1995, MNRAS, 276, 876

Delorme, P., Gagné, J., Malo, L., et al. 2012, A\&A, 548, A26
Dickey, J. M., \& Lockman, F. J. 1990, ARA\&A, 28, 215

Djorgovski, S. C., de Carvalho, R. R., Gal, R. R., et al. 1998, in New Horizons from Multi-Wavelength Sky Surveys, eds. B. J. McLean, D. A. Golombek, J. J. E. Hayes, \& H. E. Payne, IAU Symp., 179, 424

Donley, J. L., Brandt, W. N., Eracleous, M., \& Boller, T. 2002, AJ, 124, 1308

Evans, C. R., \& Kochanek, C. S. 1989, ApJ, 346, L13

Evans, P. A., Beardmore, A. P., Page, K. L., et al. 2009, MNRAS, 397, 1177

Fabbiano, G., Kim, D.-W., \& Trinchieri, G. 1992, ApJS, 80, 531

Filho, M. E., Barthel, P. D., \& Ho, L. C. 2000, ApJS, 129, 93

Gezari, S., Heckman, T., Cenko, S. B., et al. 2009, ApJ, 698, 1367

Guillochon, J., \& Ramirez-Ruiz, E. 2012, ApJ, submitted

[arXiv: 1206.2350v1]

Ho, L. C., Filippenko, A. V., \& Sargent, W. L. W. 1997, ApJS, 112, 315

Immler, S., \& Lewin, W. H. G. 2003, in Supernovae and Gamma-Ray Bursters, ed. K. Weiler, Lect. Notes Phys. (Berlin: Springer Verlag), 598, 91

Jarrett, T. H., Chester, T., Cutri, R., Schneider, S. E., \& Huchra, J. P. 2003, AJ, 125,525

Jia, J., Ptak, A., Heckman, T. M., Braito, V., \& Reeves, J. 2012, ApJ, 759, 41

Komossa, S., Halpern, J., Schartel, N., et al. 2004, ApJ, 603, L17

Lebrun, F., Leray, J. P., Lavocat, P., et al. 2003, A\&A, 411, L141

Li, L.-X., Narayan, R., \& Menou, K. 2002, ApJ, 576, 753

Lodato, G., King, A. R., \& Pringle, J. E. 2009, MNRAS, 392, 332

Lund, N., Budtz-Jørgensen, C., Westergaard, N. J., et al. 2003, A\&A, 411, L231

Marx, S., \& Pfau, W. 1992, Astrophotography with the Schmidt Telescope

Matsuoka, M., Kawasaki, K., Ueno, S., et al. 2009, PASJ, 61, 999

Merritt, D., \& Poon, M. Y. 2004, ApJ, 606, 788

Mihara, T., Nakajima, M., Sugizaki, M., et al. 2011, PASJ, 63, 623

Moshir, M., Kopan, G., Conrow, T., et al. 1990, in IRAS Faint Source Catalogue, version $2.0,0$

Nandra, K., George, I. M., Mushotzky, R. F., Turner, T. J., \& Yaqoob, T. 1997, ApJ, 476, 70

Nikołajuk, M., Czerny, B., Ziółkowski, J., \& Gierliński, M. 2006, MNRAS, 370, 1534

O'Neill, P. M., Nandra, K., Papadakis, I. E., \& Turner, T. J. 2005, MNRAS, 358, 1405

Orosz, J. A., McClintock, J. E., Aufdenberg, J. P., et al. 2011, ApJ, 742, 84

Panessa, F., Bassani, L., Cappi, M., et al. 2006, A\&A, 455, 173

Pizzella, A., Corsini, E. M., Dalla Bontà, E., et al. 2005, ApJ, 631, 785

Quanz, S. P., Goldman, B., Henning, T., et al. 2010, ApJ, 708, 770

Rees, M. J. 1988, Nature, 333, 523

Remillard, R. A., \& McClintock, J. E. 2006, ARA\&A, 44, 49

Sanders, D. B., Mazzarella, J. M., Kim, D.-C., Surace, J. A., \& Soifer, B. T. 2003, AJ, 126, 1607

Saxton, R. D., Read, A. M., Esquej, P., et al. 2012, A\&A, 541, A106

Schneider, D. P., Hall, P. B., Richards, G. T., et al. 2005, VizieR Online Data Catalog, 7243, 0

Shapley, A., Fabbiano, G., \& Eskridge, P. B. 2001, ApJS, 137, 139

Spinoglio, L., Malkan, M. A., Rush, B., Carrasco, L., \& Recillas-Cruz, E. 1995, ApJ, 453, 616

Stark, A. A., Gammie, C. F., Wilson, R. W., et al. 1992, ApJS, 79, 77

Sumi, T., Kamiya, K., Bennett, D. P., et al. 2011, Nature, 473, 349

Timmer, J., \& Koenig, M. 1995, A\&A, 300, 707

Tully, R. B., \& Fisher, J. R. 1988, Catalog of Nearby Galaxies

Ubertini, P., Lebrun, F., Di Cocco, G., et al. 2003, A\&A, 411, L131

Ulmer, A. 1999, ApJ, 514, 180

Uttley, P., McHardy, I. M., \& Papadakis, I. E. 2002, MNRAS, 332, 231

Vaughan, S., Edelson, R., Warwick, R. S., \& Uttley, P. 2003, MNRAS, 345, 1271

Véron-Cetty, M.-P., \& Véron, P. 2006, A\&A, 455, 773

Véron-Cetty, M. P., \& Veron, P. 2010, VizieR Online Data Catalog, VII/258

Walter, R., Rohlfs, R., Meharga, M. T., et al. 2010, in Eighth Integral Workshop.

The Restless Gamma-ray Universe (INTEGRAL 2010)

Walter, R., Bordas, P., Bozzo, E., et al. 2011, ATel, 3108, 1

Wang, J., \& Merritt, D. 2004, ApJ, 600, 149 